\title{
Prostatectomía radical laparoscópica. Revisión de la literatura. Nuestra experiencia
}

\author{
Cansino Alcaide JR, Álvarez Maestro M, Cabrera Castillo PM, Martínez-Piñeiro Lorenzo L, \\ Tabernero Prieto A, De la Peña Barthel JJ.
}

Servicio de Urología. Hospital Universitario La Paz. Madrid

Actas Urol Esp 2006; 30 (5): 517-530

\begin{abstract}
RESUMEN
PROSTATECTOMÍA RADICAL LAPAROSCÓPICA. REVISIÓN DE LA LITERATURA. NUESTRA EXPERIENCIA

Introducción: En éste trabajo, hemos revisado las últimas publicaciones que hacen referencia a la Prostatectomía Radical Laparoscópica (PRL) hasta el 2005 y describimos nuestra serie en éste tipo de cirugía.

Material y Métodos: Tras realizar una búsqueda en Internet, consultando revistas de reconocido prestigio, hemos seleccionado los artículos que hacen referencia a ésta técnica, resumiendo la actualidad de la PRL.

Además, presentamos nuestra serie.

Resultados: Los buenos resultados oncológicos y funcionales obtenidos con la PRL, y la posibilidad de realizar hernioplastias al igual que en cirugia abierta, hacen de la técnica, un servicio de calidad para el paciente.

Conclusiones: Los avances de la técnica, como la utilización de la robótica, cámaras de 3 CCD y la experiencia del cirujano, hacen de la PRL una técnica segura y de calidad, lo que nos obliga, en la medida de nuestras posibilidades, a ofrecerla al paciente.

Palabras clave: Laparoscopia. Cáncer de próstata. Prostatectomía radical.
\end{abstract}

ABSTRACT

ANALYSIS OF A LAPAROSCOPIC RADICAL PROSTATECTOMY PROGRAM WITH 544 CASES

Introduction: This article reviews the latest publications that refer to Laparoscopic Radical Prostatectomy (LRP) up to 2005, and describes our series of patients for this type of surgery.

Materials and methods: After a search of the Internet and consulting journals of renowned prestige, we selected articles that refer to this technique and we summarise the latest developments in LRP. We also present our series of patients.

Results: In view of the good oncologic and functional results obtained with LRP, and the possibility of performing hernioplasty as in open surgery, this technique provides a high quality service for patients.

Conclusions: Due to the advances in the safety and quality of this technique, such as the use of robots, $3 \mathrm{CCD}$ cameras, and the surgeon's experience, LRP should be offered to our patients, if it is within our means.

Keywords: Laparoscopy. Prostate cancer. Radical prostatectomy.

$\mathrm{L}^{2}$ a prostatectomía radical, está considerada como el tratamiento de referencia en el cáncer de próstata clínicamente localizado.

La técnica inicialmente descrita por Millin en 1945, fue ampliamente divulgada, a raíz de la publicación en 1983 por Walsh ${ }^{1}$.

La prostatectomía radical retropúbica, a pesar de presentar los beneficios de una cirugía extrape- ritoneal, tiene, sin lugar a dudas, los inconvenientes del dolor postoperatorio, el reducido espacio y no infrecuentemente la poca visibilidad en la pelvis durante la cirugía, lo que dificulta el control hemostático, la preservación de erectores y la realización de una correcta anastomosis vesicouretral.

En 1997 Shuessler ${ }^{2,3}$ publicó los resultados de 9 prostatectomías radicales laparoscópicas 
transperitoneales, en los que se destaca la proeza y habilidad técnica para llevar a cabo ésta cirugía. En dicha publicación, quedó patente la posibilidad técnica, aunque, el tiempo quirúrgico y las complicaciones, hicieron muy difícil su universalización.

En 1999, Gaston, Guillonneau y Vallancien ${ }^{4,5}$, describieron una serie de mejoras en la técnica, que permitieron reducir el tiempo y las complicaciones quirúrgicas.

Éste avance, permitió plantearse como real, el desarrollo de una técnica que ofreciera al paciente un postoperatorio poco doloroso, con preservación de erectores, correcta anastomosis vesicouretral, resultando en una alta tasa de continencia precoz, poco sangrado y una pronta incorporación a su actividad laboral, con la consiguiente reducción de costes, tanto hospitalarios como laborales.

En 2001, Rassweiler ${ }^{6}$ publicó la técnica retrógrada, con similares resultados iniciales.

Quedaba aún pendiente, el hecho de la universalización de la técnica y reproducibilidad de resultados, puesto que la puesta en marcha de un programa de prostatectomía radical laparoscópica, representa un esfuerzo importante en cualquier servicio de urología, por lo difícil de la técnica, larga duración de los primeros casos y la alta frecuencia de complicaciones, que en resumen se traduce en una curva de aprendizaje larga y difícil.

\section{REVISIÓN BIBLIOGRÁFICA DE LOS RESULTADOS EN PROSTATECTOMÍA RADICAL LAPAROSCÓPICA}

La prostatectomía radical laparoscópica, como toda cirugía, presenta una serie de complicaciones y dificultades técnicas, que la convierten en un reto para el urólogo en beneficio del paciente.

Hasta conseguir unos resultados comparables a cirugía abierta, tenemos que pasar por una curva de aprendizaje.

Por norma general, el número de casos necesarios para vencerla, se estima en $50-80 \operatorname{casos}^{7,8}$, a partir de los cuales, se comienza a disminuir de forma estable la duración de la cirugía, el sangrado y la tasa de complicaciones.

V. Poulakis, explica cómo consiguieron acortar la curva de aprendizaje, reduciendo drásticamente los tiempos de extracción de la pieza en sus primeros 21 casos, siendo más difícil la sutura, empleando 1,5 horas al día en simulador, seleccionando a los pacientes, visualizando los vídeos de sus propias cirugías y empleando siempre al mismo equipo de ayudantes y enfermería ${ }^{9}$.

Un dato importante respecto a la curva de aprendizaje, es la diferencia de tiempo y número de casos necesarios para vencerla, en las segundas generaciones de un mismo centro.

En estos casos, los tiempos quirúrgicos, porcentajes de transfusión, reconversión a cirugía abierta y complicaciones es mucho menor, en comparación a las primeras generaciones, lo que hace pensar, que en el futuro no haya que pasar de nuevo por las grandes dificultades iniciales, gracias a la experiencia trasmitida ${ }^{10}$.

La aplicación de la robótica, como veremos más adelante, ha tenido un papel importante en la suavización de la curva de aprendizaje.

Otro abordaje laparoscópico, es el extraperitoneal, anatómicamente similar a la prostatectomía radical retropúbica y que hasta la fecha era defendida por pocos centros.

Comunicada inicialmente por Raboy ${ }^{11}$ en 1997 y seguida por Bollens ${ }^{12}$, y Stolzengurg8 entre otros, tras acumular una experiencia previa transperitoneal de al menos 60 casos. Una vez adoptada, se reafirman y continúan con ella, argumentando a su favor, que no es técnicamente más difícil, y sin embargo, ofrece al paciente las ventajas de la cirugía mínimamente invasiva, y de la cirugía extraperitoneal, como es evitar complicaciones abdominales (ileo paralítico, accidentes intestinales...).

Brown y Dahl, tras 156 casos con experiencia extraperitoneal, presentan similares resultados de tiempo, sangrado, transfusión, márgenes positivos y complicaciones mayores, pero reconocen tener menor espacio de trabajo, más fugas de orina (aunque evitan íleos paralíticos), mayor riesgo de linfoceles si realizas linfadenectomía y evitan posibles lesiones de asas intestinales al no tener que liberar adherencias ${ }^{13}$.

Remzi y Marberger, tras 117 casos también de forma extaperitoneal, defienden éste abordaje argumentando un postoperatorio menos doloroso, y una menor tasa de estenosis de la anastomosis, menos fugas de orina y sangrado y una menor duración comparado con cirugía abierta. 
Éste artículo, presenta un comentario de Rassweiler, quien responde que en su experiencia de más de 1.200 casos, no observan claros beneficios de la técnica extraperitoneal, sobre la transperitoneal, aunque hay que formarse en ambas $^{14}$.

De todas las complicaciones, dificultades o connotaciones descritas en la literatura, las más frecuentes e importantes, y que por ello vamos a tratar son el tiempo quirúrgico, el índice de transfusión, índice de reconversión a cirugía abierta, complicaciones vasculares, digestivas, urinarias, márgenes positivos $\mathrm{y}$ resultados funcionales en cuanto a continencia y potencia.

\section{DURACIÓN DE LA CIRUGÍA}

La duración de la cirugía en la prostatectomía radical laparoscópica, es hasta la fecha y en casi todas las series publicadas, de mayor duración, debido a las dificultades de la técnica.

El hecho de que exista una gran variabilidad en las distintas series, implica la participación de distintos factores, como es la experiencia del cirujano, la compenetración con el ayudante y la experiencia de éste, y por otra parte, las dificultades del paciente; necesidad o no de realizar linfadenectomía, preservación de erectores, y volumen prostático.

El reducido espacio de trabajo en la cavidad pélvica y la mayor vascularización prostática en próstatas grandes, han sido relacionadas con mayor sangrado (Rassweiler) ${ }^{15}$. Esto implica emplear más tiempo en la hemostasia, lo que acaba por alargar la cirugía.

Indudablemente, la experiencia con prostatectomía radical laparoscópica, ha reducido el tiempo razonablemente desde las primeras series de Schussler ${ }^{2,3}$ en 1992 (9.4 horas) a las 3-4 horas de Rassweiler y Guillonneau ${ }^{15,16}$.

La técnica extraperitoneal, defendida entre otros por Stolzenburg ${ }^{8}$, presenta una duración algo menor (90-260 min) con una media de 2 horas $35 \mathrm{~min}$.

Pero es la experiencia, como demuestra Guillonneau, con la que se consigue reducir más los tiempos, llegando a pasar de $268 \mathrm{~min}$. en sus primeros 50 casos, a menos de 3 horas de los casos $450-500^{16}$.
Igualmente, Stolzenburg tras 500 casos, presenta una media de $149 \mathrm{~min}$. en pacientes sin cirugias previas ${ }^{17}$.

Rassweiler presenta una serie de prostatectomías laparoscópicas con reparación de hernias inguinales, concluyendo que la duración es algo mayor, y que ambos abordajes (transperitonealextraperitoneal) son válidos pero que de forma transperitoneal, el dolor postoperatorio es mayor debido a la necesidad de liberar peritoneo parietal, para cubrir la malla.

Destaca la especial atención sobre la estanqueidad de la sutura, para evitar el contacto de orina con la malla ${ }^{18}$.

\section{ÍNDICE DE RECONVERSIÓN A CIRUGÍA ABIERTA}

La prostatectomía radical laparoscópica es una cirugía dificil, que requiere un adecuado adiestramiento, tanto teórico como práctico en simulador y animales de experimentación, antes de dar el paso a cirugía en humanos.

Esta progresión, no es sólo por aspectos éticos sino también por la necesidad de desarrollar una habilidad y destreza técnica, necesarias para enfrentarse a la cirugía en humanos.

A pesar de ello, se describen porcentajes de reconversión a cirugía abierta, que van decreciendo a medida que se adquiere experiencia $\mathrm{y}$ número de cirugías.

En una serie publicada por Rassweiler ${ }^{15}$, se realiza una comparación de prostatectomía radical retropúbica y 2 grupos de prostatectomía radical laparoscópica de 219 pacientes cada uno, en los que se aprecia claramente, cómo con la experiencia, se mejora una serie de datos. Entre otros, el número de reconversión a cirugía abierta, siendo 8 casos en el grupo de las primeras 219 y un solo caso en el segundo grupo de 219 pacientes.

Las series que presentan una experiencia previa de al menos 70 prostatectomías radicales laparoscópicas, no suelen tener ninguna reconversión, ya que la habilidad desarrollada permite evitar accidentes o que a pesar de presentarse, se es capaz de solucionar de forma laparoscópica (lesión rectal, vascular...).

Otro factor para la reconversión, puede ser la duración de la cirugía, que naturalmente se acorta también con la experiencia. 


\section{SANGRADO Y TRASFUSIÓN}

En la prostatectomía radical laparoscópica se disfruta de una clara ventaja respecto a la cirugía abierta, que es la amplificación de la visión de los tejidos. Puede ser de 5 a 15 veces, dependiendo de la proximidad de la cámara a los mismos. Pero al mismo tiempo, pequeños sangrados son también, al igual que los tejidos, magnificados por la cámara e interfiere notablemente, no solamente en la visión directa, sino también en el efecto físico que produce la sangre en la absorción de la luz.

Por todo ello, se precisa ir controlando en todo momento el sangrado por pequeño que sea.

Otro factor que actúa en el control del sangrado, es el efecto de la presión del $\mathrm{CO}_{2}$, ya sea en cavidad peritoneal o extraperitoneal. Se trabaja habitualmente con presiones de 10-14 mmHg, que contienen el sangrado venoso, y evitan lo que sería un sangrado leve pero continuo.

Con presiones más altas, no se conseguirian grandes beneficios de contención del sangrado venoso, y por el contrario podrian aparecer complicaciones como la temida pero poco frecuente embolia gaseosa.

Por otro lado, y como es fácil de comprender, la duración de la cirugía estará relacionada en la mayoría de los casos, aunque no siempre, con el sangrado, siendo este algo mayor a mayor tiempo quirúrgico.

Rassweiller ${ }^{15}$ publicó una relación directa entre el volumen prostático y el sangrado de la siguiente manera:

- Volumen $<25$ gramos . . . . . 17\% de trasfusión.

- Volumen $>25$ gramos . . . . 47\% de trasfusión.

Eden hace referencia al tamaño de la próstata, exponiendo que a mayor edad, mayor tamaño, afectando también a la duración de la cirugía.

Describe un punto de corte sobre el tamaño, el cual hace más laboriosa la cirugía, poniéndolo en $75 \mathrm{~g}$ donde debido a un espacio mayor tras quitar la próstata, la sutura vesicouretral, puede quedar con más tensión. Además, con mayor frecuencia nos encontraremos un lóbulo medio que nos dificulta realizar una correcta preservación del cuello vesical.
También hace referencia a la anchura de pelvis en relación al tamaño prostático, que aunque en ese estudio no lo han calculado.

Nosotros también lo hemos observado y comprobamos que una pelvis ancha, te permite mayor facilidad de movimientos, a pesar de tener un elevado volumen prostático ${ }^{19}$.

En una serie publicada por Walter Artibani ${ }^{20}$, que cuestiona el beneficio de la prostatectomía radical laparoscópica frente a la cirugía abierta, presenta una serie de 50 prostatectomías abiertas realizadas por el mismo cirujano, y 71 por laparoscopia. De los 50 casos, 17 precisaron trasfusión (34\%) frente a 45 del grupo de 71 pacientes (63\%) sometidos a cirugía laparoscópica, que precisaron trasfusión, realizadas por un cirujano con experiencia previa en 60 prostatectomías radicales laparoscópicas.

En otra publicación comparativa de Rassweiler ${ }^{15}$ de cirugia abierta frente a laparoscópica, se observa cómo la pérdida de sangre es de $1.550 \mathrm{ml}$ en cirugia abierta, de $1.100 \mathrm{ml}$ en los 200 primeros casos de cirugía laparoscópica y de $800 \mathrm{ml}$ en los casi 200 segundos casos de laparoscopia, con un índice de trasfusión de 55,7\% en abierta, 30,1\% en los primeros casos de laparoscópica y de 9,6\% en el segundo grupo de casos de cirugía laparoscópica.

Evidentemente, son los primeros casos donde se presenta el mayor número de complicaciones y entre ellas, las vasculares, con lesiones de vasos epigástricos y especialmente del complejo de Santorini, lo que implica mayor sangrado. Así pues, durante los primeros 80 casos de Andrea Gregori $^{7}$, informan un 53\% de transfusión.

Guillonneau ${ }^{16}$, tras tres años de experiencia con 567 pacientes, presentó un índice de 4,9\% de transfusión, con una pérdida media de sangre de $380 \mathrm{ml}(50-1.600)$

\section{COMPLICACIONES INTRAOPERATORIAS Vasculares}

Las lesiones vasculares son, en muchas ocasiones, indicación de reconversión a cirugía abierta, fundamentalmente durante los primeros casos en los que se cuenta con poca experiencia. Una de las principales causas de lesión vascular ocurre durante la colocación de los trócares, momento en el cual se pueden ocasionar lesión de vasos epigástricos e iliacos. 
En una serie publicada por Guillonneau ${ }^{16}$, tras tres años de experiencia con 567 pacientes, presentaron 3 casos de lesión de arteria epigástrica con la colocación de los trócares que precisó control por cirugía abierta en uno de ellos.

Se recomienda siempre para evitar el riesgo de sangrado no percibido durante el tiempo quirúrgico, disminuir la presión de $\mathrm{CO}_{2}$ al final de la intervención y retirar los trócares bajo visión directa.

En caso de lesión epigástrica es recomendable no coagular y controlar mediante punto hemostático, ya sea laparoscópico o percutáneo.

Otra posibilidad de accidente vascular es el contacto directo inadvertido con pinza monopolar, fuera del campo de visión o confusión en la oscuridad del quirófano, del pedal de pinza bipolar por monopolar, cerca de estructuras vasculares.

\section{Digestivas}

Las complicaciones digestivas son fundamentalmente por íleo paralítico o por lesión de la cara anterior del recto. Ésta última puede producirse por efecto de tracción mecánica, transmisión eléctrica o sección accidental, especialmente en la técnica extrafascial.

Guillonneau tras $567 \operatorname{casos}^{16}$ presentó 1,94\% de lesión intestinal incluyendo 8 casos $(1,4 \%)$ de lesión rectal.

Rassweiler, con $438^{15}$ casos presentó 3,2\% de lesión rectal en sus primeros 219 casos y 1,8\% en sus segundos 219 , afirmándose una vez más que con la experiencia acumulada, el índice de complicaciones se reduce.

Respecto al íleo paralítico no siempre es atribuible directamente a la cirugía, como se deduce de los datos publicados por Walter Artibani ${ }^{20}$ en su serie de 71 casos de prostatectomia radical laparoscópica extraperitoneal, con un $1,4 \%$ de íleo paralítico atribuible por tanto a efectos secundarios farmacológicos (anestésicos o analgésicos).

Las lesiones intestinales debidas a tracción o perforación mecánica y transmisión eléctrica, suelen producirse de forma inadvertida fuera del campo de visión, por manipulación de pinzas sin control visual o transmisión eléctrica monopolar.

La lesión rectal es una complicación temida tanto en cirugía abierta como laparoscópica, pre- sentando unos porcentajes variables en abierta de menos de $0,5 \%$ en grandes series realizadas por un solo cirujano, como por Lepor con 1000 casos y B. Shekarriz ${ }^{21,22}$ y hasta un $9 \%$ en otras como Igel, Mc Laughlin y Borland ${ }^{23-25}$.

En las series laparoscópicas, la lesión rectal, se presenta en un 1-3\% como ha publicado Turk y Guillonneau en 125 y $1.000 \operatorname{casos}^{26,27}$, respectivamente.

Lo importante en la lesión rectal es ser capaz de detectarla intraoperatoriamente y que no pase desapercibida.

En la publicación de Guillonneau sobre el manejo laparoscópico de la lesión rectal, concluye que la mejor opción tras su experiencia con 1.000 prostatectomías laparoscópicas es suturar en 2 capas de forma continua, poniendo especial atención en reforzar el inicio y final de la sutura.

También se recomienda lavar la cavidad con antiséptico al detectar la lesión y finalizar la prostatectomía, para una vez quitada la próstata, poder suturar la lesión con mayor espacio.

Para algunos autores, puede ser de utilidad el uso de un Beniqué en el recto para ayudar a delimitar los bordes de la lesión y la estanqueidad de la sutura, o bien al finalizar ésta, rellenar la cavidad pélvica con suero e introducir aire rectal, observando burbujas de aire, en caso de cierre incompleto.

Preoperatoriamente se recomienda preparación antibiótica, pero no siempre mecánica, para prevenir infección en caso de ocurrir lesión intestinal.

En el postoperatorio se recomienda antibioterapia durante 7 días (cefalosporinas, aminoglucósidos y metronidazol), pudiendo comenzar a tolerar líquidos al día siguiente de la intervención y progresar hasta dieta normal en el quinto día postoperatorio.

La lesión rectal es más fácil cometerla durante la disección de la fascia de Denonvilliers retroapical, bien por tracción o a punta de tijera. La disección meticulosa sigue siendo la mejor prevención.

En nuestra experiencia, en los casos de pelvis estrecha y próstatas de gran tamaño, a veces es difícil por problemas de espacio, rechazar correctamente el recto a la hora de perfilar los pedículos. 
Si no se es meticuloso en éste paso, al clipar y cortar el pedículo, podemos lesionar el recto.

En los casos de lesión rectal, se ha visto en algunas series, una fuerte relación con la no preservación de erectores, posiblemente debido a la escisión amplia de los tejidos o a la falta de meticulosidad o atención que hay que prestar en los casos en los que se realiza dicha preservación, no ofrecida a veces cuando ésta no está indicada.

Así se refleja, por ejemplo, en la experiencia publicada por Guillonneau ${ }^{27}$ del manejo de las lesiones rectales. En éste artículo se informan 12 lesiones rectales en 13 pacientes a los que no se realizó preservación de erectores.

Una complicación derivada de la lesión rectal es la fístula rectouretral, publicada en varias series como Rassweiler ${ }^{15}(1,1 \%)$ durante 180 prostatectomías radicales laparoscópicas.

Cuando se reconoce la lesión rectal durante la intervención, hay que prestar especial atención a la estanqueidad de la anastomosis vesicouretral, fundamentalmente en las caras laterales y posteriores para evitar fístulas, las cuales precisan en ocasiones colostomías y sondaje uretral prolongado e incluso reparación por cirugía abierta del trayecto fistuloso.

En los casos de quemadura eléctrica, la necrosis de la pared rectal no es inmediata y debuta en el postoperatorio tardío entre los 7 y 21 días, manifestándose por pérdidas de orina por recto y/o emisión de material fecal por la orina o neumaturia.

Pero quizás más peligroso aún que la lesión rectal, sea la lesión rectal no identificada durante la cirugía.

En estos casos se produce una peritonitis, frecuentemente al $3^{\circ}-4^{\circ}$ día postoperatorio, con una presentación atípica, sin una clara reacción peritonítica, quizás algo enmascarada por la analgesia, con febrícula, ausencia de rigidez abdominal típica, sin clara leucocitosis y abdomen algo distendido.

Si ocurriera, la reintervención y colostomía con o sin sutura rectal cuando no es identificada, suele ser la norma.

\section{Urinarias}

Las lesiones ureterales suelen ser menor a un $1 \%$, tanto en cirugía abierta como laparoscópica, constituyendo una complicación poco frecuente ${ }^{16}$.
Se han descrito sección de uréter por confundirlo con el deferente ${ }^{16 .}$

Otra posibilidad de daño ureteral es durante la incisión peritoneal para movilizar la vejiga. Por ello, el límite inferior de la incisión peritoneal ha de ser el cruce del deferente.

Otra posibilidad de lesionar los uréteres, es durante la disección de las vesículas seminales y deferentes, en la técnica de Motsouris.

Una lesión no identificada durante la cirugía puede diagnosticarse en el postoperatorio por un drenaje con fuga de orina persistente y cistografía sin fuga por anastomosis.

La diseminación intraperitoneal de orina por fuga de anastomosis o lesión ureteral puede dar lugar a íleo paralítico reactivo, en los casos de cirugía transperitoneal.

\section{MÁRGENES POSITIVOS}

No hay que olvidar que se trata de una cirugía oncológica y radical, por lo que en cuanto a márgenes positivos, aun existiendo variabilidad respecto al porcentaje y localización según centros, técnica empleada, agresividad tumoral y experiencia acumulada, es en todos los casos, comparable a cirugía abierta.

El porcentaje de márgenes positivos en cirugia abierta es muy variable (5-46\%).

En series amplias como las de Lepor y Hull $^{21,28}$ con 1.000 casos, el porcentaje de márgenes positivos es del 19,9\% y $12,8 \%$ respectivamente, y en la de Sofer $^{29}$ con 734 pacientes, del $29 \%$.

Es también Lepor, quien defiende realizar prostatectomías radicales, incluso a menores de 50 años. Con 790 casos, presenta un 6,9\% de márgenes positivos global, al comparar dos grupos de mayores y menores de 50 años con cirugía abierta. Aporta unos excelentes resultados funcionales, lo cual justifica su tratamiento por su escasa morbilidad y justifica con resultados anatomopatológicos similares en ambos grupos, que los tumores de pacientes con más de 50 años, son similares anatomopatológicamente, a los de los pacientes con menos de 50 años y si tratamos a unos, debemos también tratar a los otros $^{30}$.

En la prostatectomía radical laparoscópica, también existe gran variabilidad, dependiendo de 
forma importante de la experiencia del cirujano, como lo demuestran las series publicadas por Ahmed y Tuerk ${ }^{9}$ donde 2 cirujanos con experiencia, realizan 62 prostatectomías radicales laparoscópicas y otros 2 con menos de 30 casos de experiencia, intervienen a 38 pacientes, presentando 19\% y $34 \%$ respectivamente, de márgenes positivos.

En este artículo se llega a demostrar, cómo además de la técnica empleada y la experiencia del cirujano, existen factores independientes como son el Gleason y el estadio tumoral, que influyen sobre los márgenes positivos (11\% de márgenes positivos con Gleason <7 y 50\% con Gleason 7).

La localización más frecuente de los márgenes varía según series, siendo en apex (técnica retrógrada) y posterolateral (técnica descendente) los más frecuentes.

En el grupo de Cretèil, Francia, Abbou y Salomon ${ }^{31}$ han ido modificando su técnica, en función de los resultados de márgenes positivos, obteniendo al principio en cuello, el porcentaje más amplio. Para ello modificaron su técnica, rehusando preservar el cuello, anulando así el tanto por ciento de márgenes en dicha localización.

Para disminuir el porcentaje de márgenes en apex, en 1998 comenzaron a no preservar los ligamentos puboprostáticos.

Con todo ello, presentaron en junio de 2003, su serie de 235 pacientes y 26,3\% de márgenes positivos, siendo la localización lateral la más frecuente $(41,8 \%)$ seguido de apex $(37,8 \%)$ y cuello vesical $(20,2 \%)$.

Los autores aconsejan disecar bien la uretra y esfinter para evitar los márgenes en apex y concluyen que la preservación de erectores no aumenta el riesgo de márgenes.

Otros autores como Andrea Gregori, y Alchiede Simonato ${ }^{7}$, presentan en su serie inicial de 80 casos con 5,5\% de márgenes en pT2a; 0\% pT2b; 71,4\% pT3a; 70\% pT3b; y 100\% pT4 con un global de $31,25 \%$.

Stolzenburg 8 en su serie extraperitoneal de 70 casos, presenta un total de $21,4 \%$ de márgenes (pT2 6,1\% y pT3 29,4\%) con un $52,9 \%$ de pacientes con tumor no órgano confinado.

Guillonneau, tras 1.000 casos presenta una serie de márgenes positivos: 6,9\% pT2a, 18,6\% pT2b, 30\% pT3a y 34\% en pT3b ${ }^{32}$.
Rassweiler ${ }^{15}$, presentó su serie de márgenes positivos, de forma comparativa. En cirugía abierta $28,7 \%$ en tumores órganoconfinados, frente a 21 y $23,7 \%$ en sus primeros y segundos 219 casos de prostatectomía radical laparoscópica respectivamente. Comparado con otras series abiertas, como la de Weider y Soloway ${ }^{33}$ que publicaron 28\% de márgenes positivos, demuestra que a pesar de los factores independientes como el Gleason y el estadio tumoral, la amplificación de la visión laparoscópica permite, con la adecuada experiencia, igualar e incluso mejorar series de cirugía abierta realizadas por cirujanos experimentados.

Respecto a la influencia de la preservación de erectores sobre los márgenes positivos, Palisaar y Huland, presentan unos datos que avalan la seguridad oncológica de éste aspecto técnico en la cirugía abierta, siempre y cuando se realice una correcta selección preoperartoria de los pacientes $^{34}$.

\section{RESULTADOS FUNCIONALES}

La continencia y función eréctil tras la prostatectomía radical, tanto abierta como laparoscópica, varía en función de lo que considere cada autor en los distintos trabajos.

No obstante, la continencia, que por regla general suele tener buenos resultados en cirugía abierta, se mantiene e incluso mejora en la mayoría de las publicaciones laparoscópicas.

Rassweiler ${ }^{15}$ presentó una comparativa de cirugía abierta con $89,9 \%$ de continencia a 12 meses y $93,2 \%$ a 18 meses, frente a $91,7 \%$ y $95,8 \%$ por laparoscopia a 12 y 18 meses respectivamente.

Stolzenburg 8 presentó una serie con un seguimiento de continencia muy corto con tan sólo 20 pacientes con más de 6 meses de seguimiento, de los cuales, 18 estaban completamente continentes.

Ball y D. Fabricio, observaron una alta incidencia de fugas en cara posterior en sus primeros casos, por lo que pasaron a emplear un clip de Lapra-Ty para asegurar la aproximación de la cara posterior en las 2 suturas continuas de monocryl unidas por un nudo en su extremo ${ }^{35}$.

Hofmann, ha defendido para optimizar los resultados de la continencia precoz, la intususcepción del cuello en cirugía abierta, y ciertamente lo consigue, pero a un precio quizás demasiado alto, 
ya que el daño ureteral (6/139) le obliga a realizar reimplantación ureteral en 3 ocasiones ${ }^{36}$.

Guillonneau publicó su serie de 120 casos, separándolos en 3 grupos de 40 destacando un $45 \%$ de frecuencia de erecciones con tan sólo 3 meses de seguimiento en los últimos $40 \operatorname{casos}^{37}$.

Con la llegada de la robótica, varios grupos han conseguido obtener buenos resultados de potencia, aplicando sólo control bipolar de los vasos arteriales en su entrada a la glándula, donde son más finos y más fáciles de controlar con la aplicación de menos energía.

Éste es el caso de Chien y Shalhav, quienes evitan el uso de corriente monopolar o clips y dejan que el pneumoperitoneo controle el pequeño sangrado venoso.

Definen la potencia como erecciones suficientes para penetrar y mantener relaciones con o sin fármacos vía oral y presentan un $54 \%$ de pacientes potentes a los 3 meses, $66 \%$ a los $6 \mathrm{~m}$ y $69 \%$ a los $12 \mathrm{~m}$ en una serie de 80 pacientes, de los cuales el 80\% tienen menos de 65 años.

De este artículo, destacamos que la edad media de los pacientes, es un factor primordial, a la hora de buscar buenos resultados de poten$\mathrm{cia}^{38}$.

Ahlering, Chou y Skarecky, aprovechan la robótica y describen una técnica que se basa en la disección de los pedículos y la preservación de los erectores, usando bulldog sobre el pedículo y tras cortarlo con corte frío y disecar los erectores, aplican Floseal y Gelfoam, siendo sólo preciso una ligadura, en caso de persistir sangrado arterial.

Los resultados funcionales están aún por ver, al igual que el posible efecto tóxico del Floseal sobre los erectores ${ }^{39}$.

La misma técnica de aplicar un bulldog sobre el pedículo, para evitar los clips o cualquier tipo de energías en la cercanía de los erectores, es también descrita por Gill.

Tras la sección de los pedículos, aplican ligaduras de 4/0 reabsorbibles y miden con ecodoppler transrectal, la intensidad y el número de vasos viables de los erectores antes, durante y al finalizar la cirugía.

No describen los resultados, pero hay que destacar la edad media de los pacientes de 58 años $^{40}$.

Por último, destacar tres publicaciones de prostatectomía radical laparoscópica asistida por robot, en la que Duke Herrell defiende el papel de la robótica, destacando que los buenos resultados actuales de la cirugía abierta, han alcanzado una meseta difícil de ser superados con éste abordaje, pero que la laparoscopia los ha alcanzado rápidamente, lo que hace pensar que la experiencia con la laparoscopia y más aún con la robótica, nos permita mejorar esos resul$\operatorname{tados}^{41}$.

Joseph, en la Universidad de Rochester, compara los resultados de la laparoscopia y la asistida por robot, siendo los resultados similares, pero algo mejor con la robótica en cuanto a la potencia.

Hacen hincapié en que no se debe menospreciar la formación laparoscópica previa a la utilización de la robótica. En efecto, la robótica te permite suavizar la curva de aprendizaje, pero no conseguiremos buenos resultados, si nos saltamos los pasos establecidos en la formación laparoscópica ${ }^{42}$.

Por último, el grupo de Costello en la Universidad de Melbourne, confirman la ventaja de la robótica sobre la curva de aprendizaje, al publicar su serie de 122 casos sin apenas experiencia laparoscópica previa. Obtienen unos márgenes positivos de $16,3 \%$ y una continencia del $73 \%$ en 3 meses y del $82 \%$ a los 6 meses $^{43}$.

\section{NUESTRA EXPERIENCIA}

En el servicio de Urología del Hospital Universitario La Paz, se realizan aproximadamente 150 prostatectomías radicales al año y pensamos que la cifra no disminuya en el futuro, incluso que aumente, dado que cada vez realizamos el diagnóstico más precozmente.

El programa de prostatectomía radical laparoscópica, se inició en nuestro servicio en junio de 2002, llevando realizados 544 casos en diciembre de 2005.

Casi todas las cirugias han sido realizadas de forma transperitoneal, siendo unos casos realizados mediante la técnica descendente (similar a la realizada por el Dr. Gastón en Burdeos) y otros con técnica mixta retrógrada-anterógrada (Heilbronn modificada).

Se designaron al inicio del programa a dos urólogos del servicio, con el fin de no paralizar el programa en ausencia de alguno de ellos. 
Ambos debieron realizar un entrenamiento intensivo en simulador de laparoscopia, en animales de experimentación, así como sendas rotaciones en centros con experiencia en prostatectomías radicales laparoscópicas durante 1 mes, antes de iniciar nuestro programa en humanos.

El tiempo total de entrenamiento fue de 6 meses.

Actualmente se han incorporado otros miembros del servicio como primer cirujano, incluso residentes y se prevén más incorporaciones de forma progresiva.

\section{Duración de la cirugía}

La duración media de los 544 casos es de 203,01 minutos (75-630 min) (Tabla 1).

Si separamos la duración según el número de casos realizados, comprobamos cómo con la experiencia se van acortando los tiempos, pasando de 5 horas 10 min de media en los primeros 50 casos, a las 3 horas 33 min en los casos 101147. Actualmente la duración oscila entre 90-340 (198,25 min de media) (Fig. 1).

Si lo comparamos con otros centros de referencia, observamos la misma progresión:

Guillonneau presentaba 4 horas 28 min en los primeros 50 casos y menos de 3 horas en 450$500^{13}$.

Evidentemente éste tiempo estará influenciado por la necesidad o no de realizar linfadenectomía y/o preservación de erectores.

En nuestro caso, se realizó linfadecectomía a un $15,18 \%$ y preservación de erectores en un $51 \%(26,9 \%$ bilateral). En nuestro servicio se ha realizado la revisión y estudio de 213 prostatectomías radicales retropúbicas, para obtener unas directrices generales al respecto:

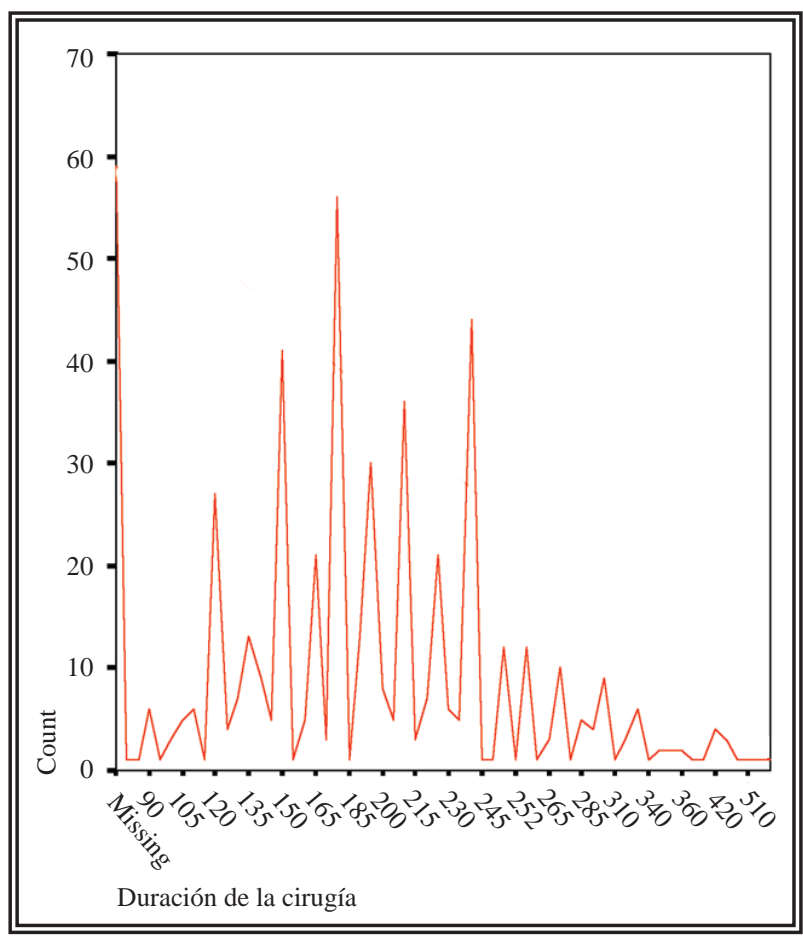

FIGURA 1. Duración de la cirugía.

Indicaciones para realizar linfadenectomía y preservación de erectores en el cáncer de próstata (Hospital Universitario La Paz)

Revisión de 213 prostatectomías radicales abiertas realizadas entre octubre de 1995 y octubre de 1999.

T1c $\mathrm{n}=106 \quad \mathrm{pN}+=3 \quad$ márgenes+ $27(24,1 \%)$

Uno de los pN+ tenía PSA $>20$, los otros dos tenían PSA<10 (uno Gleason Bx 3+4 y otro 3+3)

T2-T3a n=107 pN+=17 márgenes+37 (31,9\%)

8 de los pN+ tenían PSA $>10$ y entre los que tenían $\mathrm{PSA}<10,6$ tenían Gleason en la biopsia $>6$

Tabla 1

Descriptiva

\begin{tabular}{lcccccc}
\hline Casos & $\mathbf{1 - 5 0}$ & $\mathbf{5 1 - 1 0 0}$ & $\mathbf{1 0 1 - 1 4 7}$ & $\mathbf{1 4 8 - 2 0 0}$ & $\mathbf{2 0 1 - 2 6 2}$ & $\mathbf{2 6 2 - 5 4 4}$ \\
\hline Duración min & $300(135-630)$ & $211(105-300)$ & $210(110-330)$ & $195(110-390)$ & $181(90-420)$ & $197(105-270)$ \\
Hemorragia ml & $715(100-3500)$ & $374(100-120)$ & $389(100-1200)$ & $382(100-1200$ & $360(50-900)$ & $348(50-800)$ \\
Estancia días & $3,8(2-17)$ & $3(2-6)$ & $3(2-14)$ & $3,7(2-16)$ & $3,4(2-9)$ & $3,2(2-8)$ \\
Transfusión & $38 \%$ & $20 \%$ & $10,6 \%$ & 9,4 & $5,1 \%$ & $1 \%$ \\
\hline
\end{tabular}


Si no hubiéramos realizado linfadenectomía en casos:

- T1c + PSA<20, hubiéramos tenido 1/106 $(0,9 \%) \mathrm{pN}+$, dejando de diagnosticar 2 casos $\mathrm{pN}+(1,9 \%=2 / 106)$.

- $\mathrm{T} 1 \mathrm{c}+\mathrm{PSA}<20+$ Gleason en biopsia $<7$, hubiéramos tenido 2/106 (1,9\%) pN+, dejando de diagnosticar 1 caso $\mathrm{pN}+(0,9 \%=1 / 106)$.

- $\mathrm{T} 2-\mathrm{T} 3 \mathrm{a}+\mathrm{PSA}<10+$ Gleason en biopsia $<7$, hubiéramos tenido14/107 (13,1\%) pN+, dejando de diagnosticar 3 casos $\mathrm{pN}+$ $(2,8 \%=3 / 107)$.

Admitiendo un error diagnóstico menor del $3 \%$, podríamos obviar la linfadenectomía en los siguientes casos:

- T1c + PSA $<20+$ Gleason en biopsia $<7$

- $\mathrm{T} 2-\mathrm{T} 3 \mathrm{a}+\mathrm{PSA}<10+$ Gleason en biopsia $<7$

\section{PRESERVACIÓN DE ERECTORES}

Casos T1c

Se puede hacer preservación de erectores en los pacientes $\mathrm{T} 1 \mathrm{c}$ con $\mathrm{PSA} \leq 10$, menos de 3 biopsias positivas y sin presencia de patrón Gleason 4 ni 5 .

En estos casos la posibilidad de que el paciente tenga un tumor pT3 es del 28\% (13/46) y en ese caso la posibilidad de tener un margen positivo es del 54\% (7/13).

La posibilidad de que sea un tumor pT2 (organoconfinado) es del $72 \%(33 / 46)$ y en ese caso la posibilidad de tener un margen positivo (por incisión capsular involuntaria) es del 6\%.

La preservación de erectores se puede hacer bilateralmente, ya que cuando el tumor rebasa la cápsula prostática, en el 50\% de los casos lo hace en el lado donde las biopsias eran negativas.

\section{Casos T2-T3a}

Se puede hacer preservación de erectores en los pacientes T2-T3a con $\mathrm{PSA} \leq 10$, menos de 3 biopsias positivas y sin presencia de patrón Gleason 4 ni 5, en los cuales sólo tengan biopsias positivas en un solo lóbulo.

En estos casos la posibilidad de que el paciente tenga un tumor pT3 es del 36\% (12/33) y en ese caso la posibilidad de tener un margen positivo es del $25 \%(3 / 12)$.

La posibilidad de que sea un tumor pT2 (organoconfinado) es del 64\% (21/33) y en ese caso la posibilidad de tener un margen positivo (por incisión capsular involuntaria) es del $24 \%$.
La preservación de erectores se puede hacer en el lado sin biopsias positivas, ya que en ninguno de nuestros pacientes el tumor rebasó la cápsula en el lado contralateral al de las biopsias positivas.

\section{CONCLUSIONES}

Se pueden preservar erectores en los pacientes con $\mathrm{PSA} \leq 10$, con menos de 3 (3 de 6 o menos del 50\% de las biopsias) biopsias positivas y sin que exista ningún foco Gleason 4-5.

En los T1c pueden preservarse ambos erectores aceptando una posibilidad de márgenes positivos del $20 \%$.

En los tumores palpables T2-T3a puede preservarse el erector del lóbulo que no tenga ninguna biopsia positiva.

\section{Reconversión a cirugía abierta}

Desde Junio de 2002 a Diciembre 2005, hemos realizado 544 prostatectomias radicales laparoscópicas en las que en 4 ocasiones ha sido necesaria la reconversión a cirugía abierta.

Todos los casos se dieron dentro de las primeras 18 cirugías, lo que habla a favor de una estrecha relación con la poca experiencia en cirugía laparoscópica, puesto que en ningún otro caso posteriormente se ha precisado dicha medida.

De los 4 casos, el primero (caso 1) se debió a apertura vesical, mal control de la hemostasia y falta de experiencia.

El caso 6, a dificultad con la anastomosis.

El caso 12 presentó una hipercapnia y fibrilación auricular secundaria con imposibilidad de manejo anestésico.

En el caso 18, se provocó lesión iliaca e intestinal con la colocación de un trocar.

Todos los casos, salvo el tercero por hipercapnia, se debieron a falta de experiencia. Posteriormente se han producido situaciones similares, con dificultad en la anastomosis, control de la hemostasia, lesión rectal... que hemos solucionado de forma laparoscópica.

Al compararlo con lo publicado por otras series, todas coinciden en presentar la mayoría de las reconversiones, en sus primeros casos.

Guillonneau y Vallencien presentaron 7 reconversiones en 567 casos $^{13}$, siendo todos en los primeros 70 casos. 
Rassweiler publicó 9 de $438^{12}$, siendo un solo caso, posterior a los primeros 219 .

\section{Sangrado y transfusión}

Las pérdidas sanguíneas anotadas en nuestros 544 casos, dibujan una línea descendente, con $348 \mathrm{ml}$ de media (50-3.500) y $7 \%$ de transfusión. (Fig. 2).

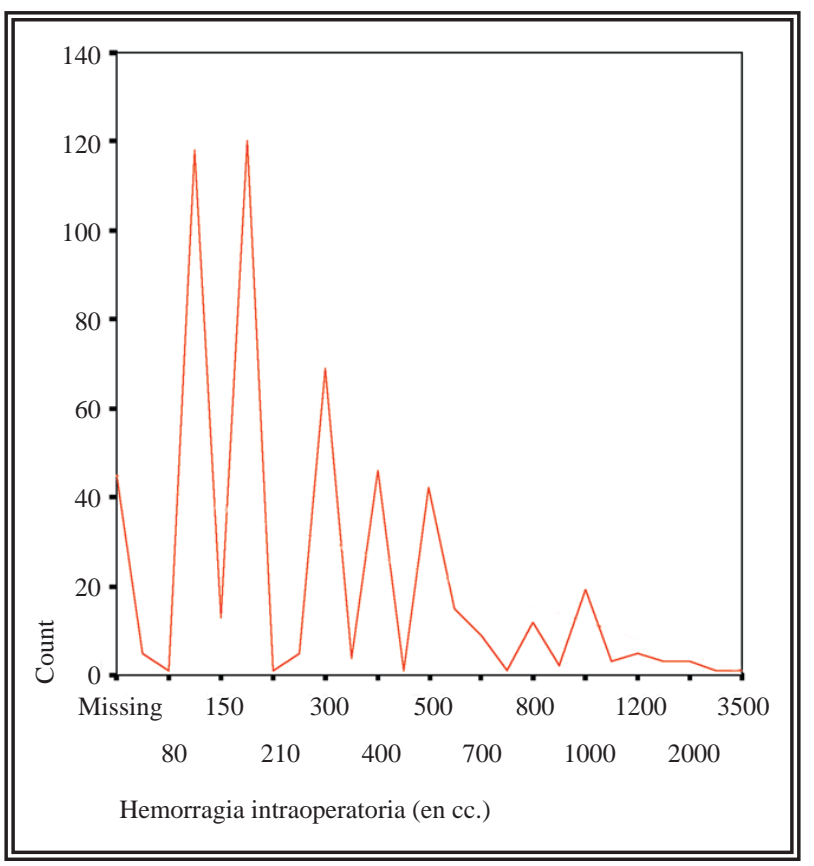

FIGURA 2. Hemorragia intraoperatoria.

Comparando los primeros 50 casos $(750 \mathrm{ml} \mathrm{de}$ media y $37 \%$ de transfusión) con los casos 101147 (374 ml y 10.6\% de transfusión), observamos dicha progresión.

Igualmente, el porcentaje de transfusión disminuye de $58,3 \%$ a $10,7 \% 5,1 \%$ y $1 \%$ en los años 2002, 2003, 2004 y 2005.

Si lo comparamos con otros centros, observamos la misma tónica:

Rassweiler ${ }^{12}$ publicó $1.100 \mathrm{ml}$ en sus primeros 219 casos y $800 \mathrm{ml}$ posteriormente.

Stolzenburg 8 presenta una media de $350 \mathrm{ml}$ (100-850) y un $1,4 \%$ de transfusión, en 70 casos realizados de forma extraperitoneal, con una experiencia previa transperitoneal de 70 casos.

Guillonneau ${ }^{13}$ publicó una pérdida de $380 \mathrm{ml}$ (50-1600) y 4,9\% de transfusión, siendo en los primeros 50 casos, $514 \mathrm{ml}$ de media y de $292 \mathrm{ml}$ en los casos 500-567.

\section{Complicaciones intraoperatorias}

Durante las 544 prostatectomías realizadas hasta Diciembre 2005, se han podido rescatar los datos de 476 pacientes para complicaciones Intraoperatorias y 494 de complicaciones postoperatorias que se describen a continuación en las Tablas 2 y 3.

Tabla 2

Complicaciones intraoperatorias recogidas sobre 476 pacientes

\begin{tabular}{lcc}
\hline Complicaciones intraoperatorias & $\begin{array}{c}\text { No de } \\
\text { casos }\end{array}$ & \% \\
\hline Lesion vascular mayor & 2 & $0,42 \%$ \\
Apertura vesical & 3 & $0,63 \%$ \\
Apertura rectal & 11 & $2,31 \%$ \\
Hemorragia & 3 & $0,63 \%$ \\
Hemorragia, dificultad anastomosis & 2 & $0,42 \%$ \\
Lesión arteria epigástrica & 1 & $0,21 \%$ \\
Apertura trígono en línea media & 2 & $0,42 \%$ \\
Dificultad anastomosis, desgarro uretra & 4 & $0,84 \%$ \\
Dificultad disec vesiculas, disec retrograda & 1 & $0,21 \%$ \\
Apertura cúpula vesical & 2 & $0,42 \%$ \\
Neumotórax a tensión & 1 & $0,21 \%$ \\
Enfisema subcutáneo & 1 & $0,21 \%$ \\
Herniorrafia inguinal & 2 & $0,42 \%$ \\
Estenosis uretra, falsa via, sondaje bajo vision & 1 & $0,21 \%$ \\
Zona posterior de anastomosis separada & 1 & $0,21 \%$ \\
\hline
\end{tabular}

Tabla 3

Complicaciones postoperatorias recogidas sobre 494 pacientes

\begin{tabular}{lcc}
\hline Complicaciones postoperatorias & $\begin{array}{c}\text { No de } \\
\text { casos }\end{array}$ & $\%$ \\
\hline Hemorragia que requiere transfusion & 14 & $2,83 \%$ \\
Hemorragia que requiere cirugia laparoscópica & 2 & $0,4 \%$ \\
Hemorragia que requiere cirugia abierta & 2 & $0,4 \%$ \\
Sospecha de lesión intraabd. que requiere cirugia & & \\
abierta & 2 & $0,4 \%$ \\
Hematuria & 4 & $0,8 \%$ \\
Distension abdominal sintomática -ileoparalítico & 13 & $2,63 \%$ \\
Fuga de orina & 6 & $1,21 \%$ \\
IRA + ileo paralitico & 1 & $0,2 \%$ \\
Sangrado peridrenaje & 1 & $0,2 \%$ \\
Gastroenteritis & 7 & $1,41 \%$ \\
Se arranca la sonda & 1 & $0,2 \%$ \\
Neuropatia periferica & 1 & $0,2 \%$ \\
Dolor perineal & 1 & $0,2 \%$ \\
Hernia trócar 10 & 1 & $0,2 \%$ \\
Linforrea & 1 & $0,2 \%$ \\
TVP & 1 & $0,2 \%$ \\
Infeccion herida & 2 & $0,4 \%$ \\
Hernia inguinal estrangulada & 1 & $0,2 \%$ \\
Diarrea sanguinolenta & 1 & $0,2 \%$ \\
Lesion rectal, lesion ileal, Fournier & 1 & $0,2 \%$ \\
Absceso pelviano y fiebre & 1 & $0,2 \%$ \\
Fistula rectouretral o rectovesical & 1 & $0,2 \%$ \\
\hline & &
\end{tabular}




\section{Márgenes positivos}

Si comparamos los márgenes positivos globales de las prostatectomías radicales retropúbicas con las laparoscópicas, observamos que no hay grandes diferencias:

pT2: $19 \%(73 / 385)$ en laparoscopia y $16,8 \%$ (21/125) en abierta y pT3: 53,8\% (78/145) laparoscópica y $51,1 \%(45 / 88)$ en abierta.

La localización de los márgenes, puede estar relacionada, como hemos mencionado anteriormente, con la técnica empleada y la experiencia del cirujano.

Tenemos que tener en cuenta, que los casos revisados de prostatectomía radical abierta, se operaron tras contar con más de 10 años de experiencia con la técnica, mientras que la experiencia acumulada con prostatectomía radical laparoscopia en nuestro servicio no llega a los 4 años.

En nuestra serie, la localización más frecuente es posterolateral unilateral con 50 casos (50/133 37,5\%), seguido de ápex unilateral con $25(25 / 13318,7 \%)$.

Hay cierta discrepancia en la literatura sobre la incidencia de márgenes positivos, en aquellos casos en los que se realiza preservación de erectores. Algunos autores refieren menor tasa de márgenes positivos, en los casos que se preservan erectores. Es posible que se debido en parte a la selección de $\operatorname{casos}^{9}$. En nuestra experiencia, la tasa de márgenes no se ve afectada negativamente en los casos de preservación de erectores.

Así, en nuestra serie, tenemos un $31,3 \%$ de márgenes positivos en los casos en los que no se realiza preservación de erectores, $28,1 \%$ si se realizó preservación unilateral y $29,6 \%$ si se preservó de forma bilateral.

Comparando nuestra serie en cirugía abierta revisada junto con la laparoscópica, con las distintas series de otros centros de referencia publicadas, podemos decir, que nuestros porcentajes de márgenes positivos son similares ${ }^{8,12,26}$.

\section{Resultados funcionales}

Nuestra serie es todavía relativamente corta en el tiempo, como para poder presentar un número importante de casos con más de 12 meses de seguimiento.
Además, estos casos son los primeros y por la poca experiencia del momento, no son comparables a los últimos respecto a la calidad de la preservación del cuello vesical, esfinter uretral y haces neurovasculares.

De los 283 pacientes con datos completos recogidos y más de 12 meses de seguimiento, el $80,7 \%$ presentan perfecta continencia o usan una compresa de seguridad (17\%). Si valoramos solo los últimos 50 casos de éstos 283 , tenemos un $86 \%$ de buenos resultados (no protección o una pequeña compresa de seguridad al día). A 24 meses de seguimiento este porcentaje asciende a 91,4\% (81 pacientes).

En cuanto a la función eréctil, de los 16 pacientes con más de 24 meses de seguimiento con preservación bilateral, $6(37,5 \%)$ mantiene relaciones sexuales (con penetración) con o sin necesidad de medicación oral. Teniendo en cuenta que la edad media de nuestros pacientes está por encima de los 60 años $(64,15)$, nuestros resultados son comparables a los publicados por Mani Menon en éste rango etario ${ }^{44}$.

Otros datos de interés son una estancia media de 3,4 días (2-44) y 16,5 días con sonda (7-60) (Figs. 3 y 4).

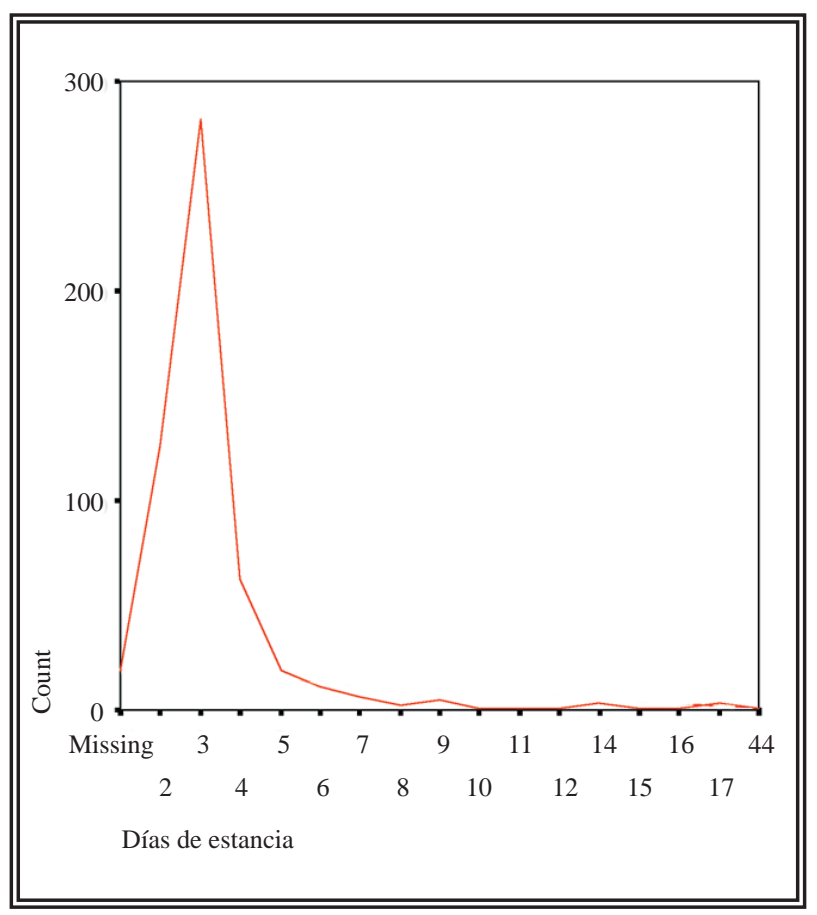

FIGURA 3. Estancia postoperatoria. 


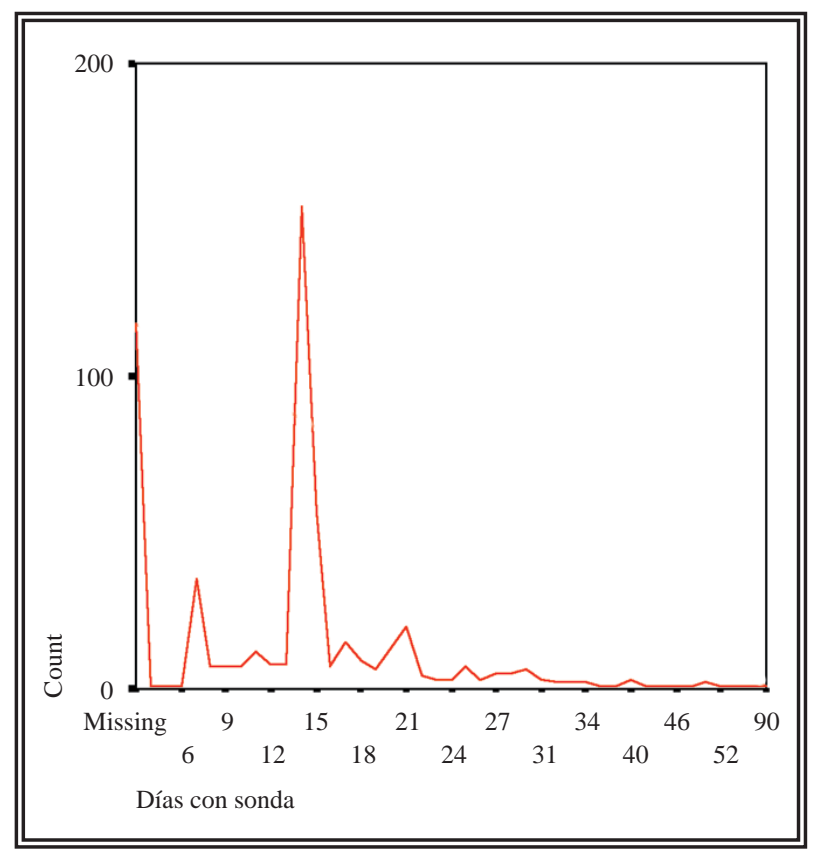

FIGURA 4. Dias con sonda.

El tiempo de sondaje se está muy influido por la imposibilidad de realizar cistouretrografías a todos los pacientes, dado el elevado número de enfermos que operamos. Durante varios meses realizamos cistouretrografias a todos los enfermos a los 7 días y comprobamos que aproximadamente el $50 \%$ de los pacientes tenía una fuga de contraste en la anastomosis. A partir de ese momento decidimos, como política del Servicio, el retirar la sonda a todos los pacientes a los 14 días, sin realizar cistouretrografias previas.

En una escala analógica para el dolor, con puntuación de 0-10, se recoge éste dato al segundo día de postoperatorio, siendo la media de $2(0-8)$.

Agradecimientos: Agradecemos la colaboración de los Dres. Alonso Dorrego y López-Tello en la revisión de las historias de los pacientes, así como a los demás miembros del servicio de urología por remitirnos a los enfermos.

\section{REFERENCIAS}

1. Walsh PC, Lepor H, Eggleston JC. Radical prostatectomy with preservation of sexual funtion: anatomical d pathological considerations. Prostate. 1983;4(5):473-85

2. Schuessler WW, Kavoussi LR, Clayman RV, Vancaillie TG. Laparoscopic radical prostatectomy: initial case report. J Urol 147 (1992), p.246A (Abstract 130).
3. Schuessler WW, Schulamn PG, Clayman RV, Kavoussi LR. Laparoscopic radical prostatectomy: initial short-term experience. Urology 1997;50(6):854-857.

4. Guillonneau B, Cathalineau X, Barret E, Rozet F. Vallancien G. Laparoscopic radical prostatectomy. Preliminary evaluation after 28 interventions. Presse Med. 1998; 27(31):15701574.

5. Guillonneau B, Cathalineau X, Barret E, Rozet F, Vallancien G, Laparoscopic radical prostatectomy: technical and early oncological assessment of 40 operations Eur Urol. 1999;36(1):14-20.

6. Rassweiler J, Sentker L, Seeman O, Hatzinger M, Stock C, Frede T. Heilbronn Laparoscopic radical prostatectomy. Eur Urol. 2001;40(1):54-64.

7. Gregori A, Simonato A, Lissiani A, Bozzola A, Galli S, Gaboardi F. Laparoscopic radical prostatectomy: perioperative complications in an initial and consecutive series of 80 cases. Eur Urol. 2003;44(2):190-194.

8. Stolzenburg JU, Do M, Rabenalt R, Pfeiffer H, Horn L, Truss MC, Jonas U, Dorschner W. Endoscopic extraperitoneal radical prostatectomy: initial experience after 70 procedures. J Urol. 2003;169(6):2066-2071.

9. Vassilis Poulakis, Wolfgang Dillenburg, Matthias Moeckel, Rachelle de Vries, Ulrich Witzsch, Jean Rassweiler, et al. Laparoscopic Radical Prostatectomy: Prospective evaluation of the learning curve. Eur Urol. 2005;47:167-175.

10. El-Feel A, Davis JW, Deger S, Roigas J, Wille AH, Schnorr $\mathrm{D}$, et al. Positive margins after laparoscopic radical prostatectomy: a prospective study of 100 cases performed by 4 different surgeons. Eur Urol. 2003;43(6):622-626.

11. Raboy A, Ferzli G, Albert P. Initial experience with extraperitoneal endoscopic radical retropubic prostatectomy. Urology. 1997;50(6):849-853.

12. Bollens R, Vanden Bossche M, Roumeguere T, Damoun A, Ekane S, Hoffmann P, et al. Extraperitoneal laparoscopic radical prostatectomy. Results after 50 cases. Eur Urol. 2001 Jul;40(1):65-69.

13. Brown JA, Rodin D, Lee B, Dahl DM. Transperitoneal versus Extraperitoneal approach to laparoscopic radical prostatectomy: an assessment of 156 cases. Urology. 2005; 65(2):320-324.

14. Remzi M, Klingler HC, Tinzl MV, Fong YK, Lodde M, Kiss B, et al. Morbidity of laparoscopic extraperitoneal versus transperitoneal radical prostatectomy versus open retropubic radical prostatectomy. Eur. Urol. 48(2005) 83-89.

15. Rassweiler J, Sentker L, Seemann O, Hatzinger M, Rumpelt HJ. Laparoscopic radical prostatectomy with the Heilbronn technique: an analysis of the first 180 cases. J Urol. 2001;166(6):2101-2108.

16. Guillonneau B, Rozet F, Cathalineau X, Lay F, Barret E, Vallancien G. Perioperative complications of laparoscopic radical prostatectomy: The Montsouris 3-year experience. J Urol. 2002;167(1):51-56

17. Stolzenburg JU, Ho KM, Do M, Rabenalt R, Dorschner W, Truss MC. Impact of previous surgery on endoscópic extraperitoneal radical prostatectomy Urology. 2005;65(2): 325331 .

18. Teber D, Erdogru T, Zukosky D, Frede T, Rassweiler J. Prosthetic mesh hernioplasty during laparoscopic radical prostatectomy. Urology. 2005;65(6):1173-1178.

19. Chang CM, Moon D, Gianduzzo TR, Eden CG. The impact of prostate size in laparoscopic radical prostatectomy. Eur Urol. 2005;48(2):285-290. 
20. Artibani W, Grosso G, Novara G, Pecoraro G, Sidoti O, Sarti A, et al. Is laparoscopic radical prostatectomy better than traditional retropubic radical prostatectomy Eur Urol. 2003 Oct;44(4):401-406.

21. Lepor H, Nieder AM, Ferrandino MN. Intraoperative and postoperative complications of radical retropubic prostatectomy in a consecutive series of 1,000 cases $\mathrm{J}$ Urol 2001;166(5):1729-1733.

22. Shekarriz B, Updhyay J, Wood DP. Intraoperative, perioperative and long-term complications of radical prostatectomy. Urol Clin North Am. 2001;3:639-653.

23. Igel TC, Barrett DM, Segura JW, Benson RC Jr, Rife CC Perioperative complications from bilateral pelvic lymphadenectomy and radical retropubic prostatectomy. J Urol. 1987 Jun;137(6):1189-1191

24. McLaughlin AP 3rd, McCullough DL. Successful urologic management of inadvertent rectal injuries. J Urol. 1971 106(6):878-880.

25. Borland RN, Walsh PC. The management of rectal injury during radical retropubic prostatectomy. J Urol. 1992;147 (3 Pt 2):905-907.

26. Turk I, Deger S, Winkelmann B, Schonberger B, Loening SA. Laparoscopic radical prostatectomy. Technical aspects and experience with 125 cases. Eur Urol. 2001;40(1):4652 .

27. Guillonneau B, Gupta R, El Fettouh H, Cathelineau X, Baumert H, Vallancien G. Laparoscopic management of rectal injury during laparoscopic radical prostatectomy. J Urol. 2003;169(5):1694-1696.

28. Hull GW, Rabbani F, Abbas F, Wheeler TM, Kattan MW Scardino PT. Cancer control with radical prostatectomy alone in 1,000 consecutive patients. J Urol. 2002;167(2 Pt 1): 528-534.

29. Sofer M, Hamilton-Nelson KL, Civantos F, Soloway MS., Positive surgical margins after radical prostatectomy: The influence of site and number on progression. J Urol. 2002; 167(6):2453-2456.

30. Twiss C, Slova D, Lepor H. Outcomes for men younger than 50 years undergoing radical prostatectomy. Urology. 2005;66(1):141-146.

31. Katz R, Salomon L, Hoznek A, de la Taille A, Antiphon P, Abbou CC. Positive surgical margins in laparoscopic radical prostatectomy: The impact of apical dissection, bladder neck remodeling and nerve preservation. J Urol. 2003;169 (6):2049-2052 .

32. Guillonneau B, el-Fettouh H, Baumert H, Cathelineau X, Doublet JD, Fromont G, et al. Laparoscopic radical prostatectomy: Oncological evaluation after 1.000 cases at Montsouris Institute J Urol. 2003;169(4):1261-1266.
33. Wieder JA, Soloway MS. Incidence, etiology, location, prevention and treatment of positive surgical margins after radical prostatectomy for prostate cancer. J Urol. 1998; 160(2):299-315.

34. Palisaar RJ, Noldus J, Graefen M, Erbersdobler A, Haese A, Huland $H$. Influence of nerve-sparing (ns) procedure during radical prostatectomy on margin status and biochemical failure. Eur Urol. 2005;47(2):1761-1784.

35. Ball AJ, Bordeau KP, Davis JW, Given RW, Lynch DF, Fabrizio MD. Modified running vesicourethral anastomosis after robotically assisted laparoscopic radical prostatectomy: use of solitary Lapra-Ty to secure posterior approximation. Urology. 2005;66(1):16-18.

36. Wille S, Varga Z, von Knobloch R, Hofmann R. Intussuscepction of bladder neck improves early continence after radical prostatectomy: results of a prospective trial. Urology. 2005;65(3):524-527.

37. Guillonneau B, Vallancien G. Laparoscopic radical prostatectomy: the Montsouris technique. J Urol. 2000;163(6): 1643-1649.

38. Chien GW, Mikhail AA, Orvieto MA, Zagaja GP, Sokoloff $\mathrm{MH}$, Brendler CB, et al. Modified clipless antegrade nerve preservation in robotic-assisted laparoscopic radical prostatectomy with validaded sexual function evaluation. Urology. 2005;66(2):419-423.

39. Ahlering TE, Eichel L, Chou D, Skarecky DW. Feasibility study for robotic radical prostatectomy cautery-free neurovascular bundle preservation. Urology. 2005;65(5):994-997.

40. Gill IS, Ukimura O, Rubinstein M, Finelli A, Moinzadeh A, Singh D, et al. Lateral pedicle control during laparoscopic radical prostatectomy: refined technique.Urol. 2005;65: 23-27.

41. Herrell SD, Smith JA Jr. Laparoscopic and robotic radical prostatectomy: what are the real advantages? BJU Int. 2005;95(1):3-4.

42. Joseph JV, Vicente I, Madeb R, Erturk E, Patel HR. Robotassisted vs pure laparoscopic radical prostatectomy: are there any differences?. BJU Int. 2005;96(1):39-42.

43. Costello AJ, Haxhimolla $\mathrm{H}$, Crowe $\mathrm{H}$, Peters JS. Installation of telerobotics surgery and inicial experence with telerobotics radical prostatectomy. BJU Int. 2005;96: 3438.

44. Tewari A, Peabody J, Searle R, Balakrishnan G, Hemal AK, Shrivastaba A, et al. Technique of da Vinci robot assisted anatomic radical prostatectomy. Urology. 2002;60(4):569-572.

Dr. J.R. Cansino Alcaide

E-mail: urocansino@yahoo.es 